\title{
Genetic Algorithm Optimal Design on Diaphragm Spring by MATLAB
}

\author{
Shuxin Chen ${ }^{1, a}$, Lingyu Zhang ${ }^{1, b}$ and Xingwang Cheng ${ }^{2, c}$ \\ ${ }^{1}$ Computer Center, Qiqihar University, Heilongjiang Qiqihar 161006, China \\ ${ }^{2}$ School of Mechanical Engineering ,Qiqihar University, Heilongjiang Qiqihar 161006, China \\ a shuxin@126.com, ${ }^{b}$ zhangling@126.com, ${ }^{c}$ 45102@qq.com
}

Keywords: Clutch; Diaphragm Spring; Reserve Coefficient; Optimized Design

\begin{abstract}
Diaphragm spring is one of the key parts of the clutch. The paper set up a mathematical model to optimize its parameters, which can be make reserve coefficient stability, improve clutch reliability, through analyzing its deformation-load characteristic curve. Based on an instance, the results showed that the optimal design parameters were significantly better than the original design.
\end{abstract}

\section{Introduction}

We can find the diaphragm spring has nice non-linear characteristics by comparing with the traditional coil spring clutch. Using it in the clutch could simplify the structure greatly, so that the clutch reaches to utterly compact on assembly, and transmit the more engine torque than the coil. As the optimization enhancing and manufacture processing, the diaphragm spring has been getting wonderfully expand. However their conditions needed thinking are more and more complex. Through using the genetic algorithm (GA) to optimize the parameters of the original design, we can get a satisfied result expectedly. Before the friction disc becomes ineffectiveness. Make it, what is pressing force on the diaphragm, changes reach to minimum. On the other hand, after the plate invalid, the clutch still delivery the engine torque and the force changes in a small areas.

The diaphragm spring structure and deformation-load equation

The diaphragm spring construction as shown above, Diaphragm fingers and disc spring portion make up the formation[1]. The specific parameters include: the outer radius of diaphragm spring $\mathrm{R}$, Inner radius of disc $r$, height of disc spring $H$, Thickness of diaphragm spring $h$, conical bottom angle $\alpha$, Inner radius of diaphragm $r_{0}$, radius of diaphragm finger in the widest point $r_{e}$, The width of the incision in separated finger partition, Maximum width of slotting,Load action radius of the clutch cover $R_{1}$,Pressure plate bearing action point radius $r_{1}$, and the number of fingers $n$.

The diaphragm spring shaped-load equation. While the clutch deformed, as the A-L formula is shown, the relationship between the outer loaded $P_{1}$ and deformation $\lambda$ is:

$$
P_{1}(\lambda)=\frac{\pi E h \lambda \ln \frac{R}{r}}{6\left(1-\mu^{2}\right)\left(R_{1}-r_{1}\right)^{2}}\left\{\left(H-\lambda \frac{R-r}{R_{1}-r_{1}}\right)\left(H-\frac{\lambda}{2} \cdot \frac{R-r}{R_{1}-r_{1}}\right)+h^{2}\right\}
$$

Where $E$ is the modulus of elasticity (MPa), For the steel $E=2.1 \times 105 ; \mu$ is Poisson ratio, the value is 0.3 . As the figure 2 is shown. Based on the third strength theory[2], $\mathrm{A}$ is the maximum equivalent stress point. We can deduce its calculated formula:

$$
\sigma_{\mathrm{V} B}=\frac{E}{1-\mu^{2}} \cdot \frac{\lambda}{R_{1}-r_{1}}\left[\left(\frac{R-r}{r \ln \frac{R}{r}}-1\right)\left(\frac{H}{R-r}-\frac{\lambda}{2\left(R_{1}-r_{1}\right)}\right)+\frac{h}{2 r}\right]-\frac{6 P_{2}\left(r-r_{f}\right) E}{\pi r \beta h^{2}}
$$

Where $\beta$ is a coefficient of the root of separation fingers' width and the value is:

$$
\beta=1-\frac{\delta_{2} \cdot n}{\pi\left(r_{e}+r\right)}
$$



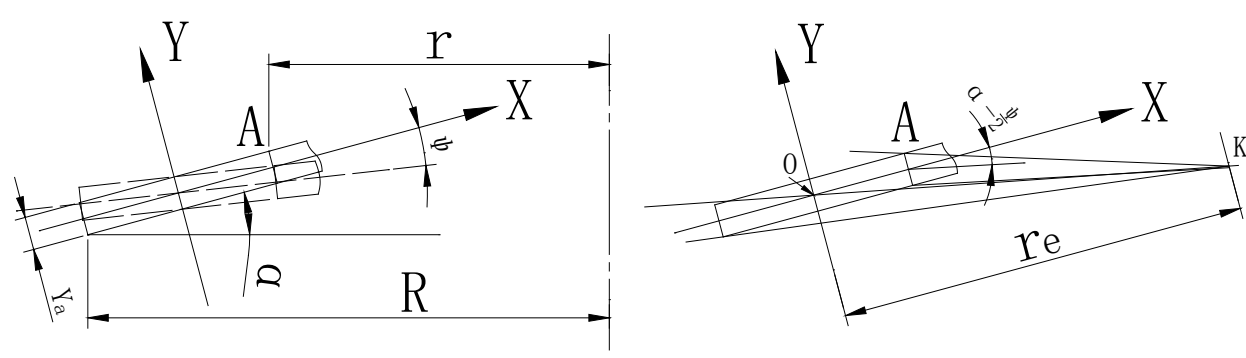

\section{Genetic Algorithms}

Fig 1 Stress analysis and distribution of diaphragm spring

Based on Evolution and Genetics, Genetic algorithm, which originated in simulating the mechanisms what are the study of biological systems, is a methods, which is solving global optimization. This algorithm starts as populations that containing the solution set. Then, there will be formatting a number of individual in each internal population through genetic coding. One thing, however, is certain: each individual corresponds to a chromosome with the gene coding. Next, Many excellent individual, through chromosomal chiasma and mutation which is hearly impossible to determine, pass their genes, that according to the size of the fitness value update and it's higher than the last generation. It will be not stopped until the conclusions satisfied. Finally, By comparing the fitness value in parallel of individual of each population and selecting the best one returning it to the user[3].

Diaphragm spring working point . As the spring deformation-load characteristic curve shown, we can get it, that $\mathrm{B}$ is a new diaphragm operating point in the clutch generally from fig 1 . the formation at B which is the working point of pressure condition generally taken $\left(0.7^{\sim} 0.8\right) H$. With the fiction disk is constantly wear and tear at work, the point will be changed from B to A, as soon as it failed. And the distance is:

$$
\Delta \lambda=Z \Delta S_{0}=\lambda_{B}-\lambda_{A}
$$

Where $Z$ is a number of working face clutch friction plate. For the single plate $Z=2 . \Delta S_{0}$ is the maximum amount of wear and tear on one side of the clutch friction plate, generally ranges is from $0.65 \mathrm{~mm}$ to $1.1 \mathrm{~mm}$.

When the clutch separate completely. The distance where the separation point $C$ to the normal point $B$ calculation formula is is:

$$
\lambda_{f}=Z \Delta S
$$

Where $\Delta S$ is a gap between the clutch friction plate separated fully and the install new spring's formation. For the single generally take $0.75 \mathrm{~mm}$ to $1 \mathrm{~mm}$.

Optimal design model of the diaphragm spring.Based on the Eq.1, we can obtain the independently main parameters $\mathrm{H}, \mathrm{h}, \mathrm{R}, \mathrm{r}, \mathrm{R}_{1}, \mathrm{r}_{1}$ six variables, and the mathematical model is[4]:

$$
\begin{gathered}
\operatorname{Min} F(x)=\omega_{1} f_{1}(x)+\omega_{2} f_{2}(x) \\
\left\{\begin{array}{c}
f_{1}(x)=\operatorname{Min} \frac{1}{5} \sum_{i=1}^{5}\left|P_{1}\left(x_{1}, x_{2}, x_{3}, x_{4}, x_{5}, x_{6}, \lambda-\frac{i}{5} \Delta \lambda\right)-P_{1}\left(x_{1}, x_{2}, x_{3}, x_{4}, x_{5}, x_{6}, \lambda\right)\right| \\
f_{2}(x)=\operatorname{Min}\left|P_{1}\left(x_{1}, x_{2}, x_{3}, x_{4}, x_{5}, x_{6}, \lambda-\lambda_{f}\right)-P_{1}\left(x_{1}, x_{2}, x_{3}, x_{4}, x_{5}, x_{6}, \lambda\right)\right| \\
x=\left[x_{1}, x_{2}, x_{3}, x_{4}, x_{5}, x_{6}\right]^{T}=\left[H, h, R, r, R_{1}, x_{1}\right]^{T}
\end{array}\right.
\end{gathered}
$$

According to their affects, make their weight coefficient is 0.7 and 0.3 , respectively . 


\section{Constraints}

(1)The diaphragm spring load-deformation characteristic curve is gratefully impacted by thickness ratio, the ratio should meet a certain range:

$$
1.5 \leq H / h \leq 2
$$

(2) The ratio of the relative size of each portion of the diaphragm spring within a predetermined range:

$$
\begin{aligned}
& 1.20 \leq R / r \leq 1.35 \\
& 70 \leq 2 R / h \leq 100 \\
& 3.2 \leq R / r_{0} \leq 5
\end{aligned}
$$

(3) To make clamping force distributed uniformly on the clutch friction plate, the values pressure plate loading point radius should be in the radius of the friction plate and the friction plate diameter, namely:

$$
\frac{D+d}{4} \leq R_{1} \leq \frac{D}{2}
$$

(4) While the diaphragm spring in the free state, the diaphragm spring need to meet performance which is the requirement of the clutch. Its initial cone angle needs to be within a certain range of values, that according to the relationship between the corner and the height.

$$
9^{\circ} \leq \alpha=\arctan \frac{H}{R-r} \leq 15^{\circ}
$$

(5) Based on spring structural layout requirements, 1 to 2, 3 to 4, 5 to 6 should be within a certain range usually:

$$
\begin{aligned}
& 1 \leq R-R_{1} \leq 7 \\
& 0 \leq r_{1}-r \leq 6 \\
& 0 \leq r_{f}-r_{0} \leq 4
\end{aligned}
$$

(6) The diaphragm spring is not only working in action of the compression spring, but also playing a role of the separation device. So its leverage ratio should be selected within a certain range:

$$
3.5 \leq \frac{r_{1}-r_{f}}{R_{1}-r_{1}} \leq 9.0
$$

(7) To ensure the strength of the conditions within the allowable range, avoid fatigue fracture failure, and the stress need to meet:

$$
\sigma_{\mathrm{VB}} \leq\left[\sigma_{V B}\right]
$$

\section{Examples}

Based on a single light vehicle models pull-type structure parameters of diaphragm spring clutch for the design. The material of diaphragm spring is $\mathrm{Si}_{2} \mathrm{M}_{\mathrm{n}}$, and its allowable stress is $1700 \mathrm{Mpa}$. The primary structure parameters are: $\mathrm{H}=3.5 \mathrm{~mm}, \mathrm{~h}=2 \mathrm{~mm}, \mathrm{R}=83 \mathrm{~mm}, \mathrm{r}=67.5 \mathrm{~mm}, \mathrm{R}_{1}=78 \mathrm{~mm}, \mathrm{r}_{1}$ $=68.5 \mathrm{~mm}$, and the release bearing action radius $\mathrm{rf}=26 \mathrm{~mm}$, the displacement of the spring operating points relatively is: $\lambda_{\mathrm{B}}=2.6 \mathrm{~mm} ; \Delta S_{0}=0.9 \mathrm{~mm} ; \Delta S=1 \mathrm{~mm}$. In this case assign the initial population 100 , the termination of algebra is 100 , the crossover probability is 0.8 , the mutation probability 0.01 . Using the above parameters and object function to optimize the design and the result compared is shown in Table 1. Drawing the deformation - load character curve of the diaphragm shown in Fig 2.

It's not difficult to find, there is needed smaller power than before. Separation handles performance and the reserve-coefficient becomes stable. Optimized diaphragm spring in the process of the pressure wear and tear had been improved. Both indicators have been adjusted in the aspects of the maximum force and the equivalent stress at the maximum stress point, after calculating decreased $10.66 \%$ and $22 \%$, respectively. 
Table 1 Comparison of the parameters before and after optimization design

\begin{tabular}{c|c|c}
\hline Parameters & Original design & optimal design \\
\hline$H / m m$ & 3.5 & 3.5 \\
\hline$h / m m$ & 2 & 2 \\
\hline$R / m m$ & 83 & 80.01 \\
\hline$r / m m$ & 67.5 & 62.12 \\
\hline$R_{1} / m m$ & 78 & 76.41 \\
\hline$r_{1} / m m$ & 68.5 & 65.21 \\
\hline Pmax & 5355 & 4784 \\
\hline stress & 1051 & 821.1 \\
\hline
\end{tabular}

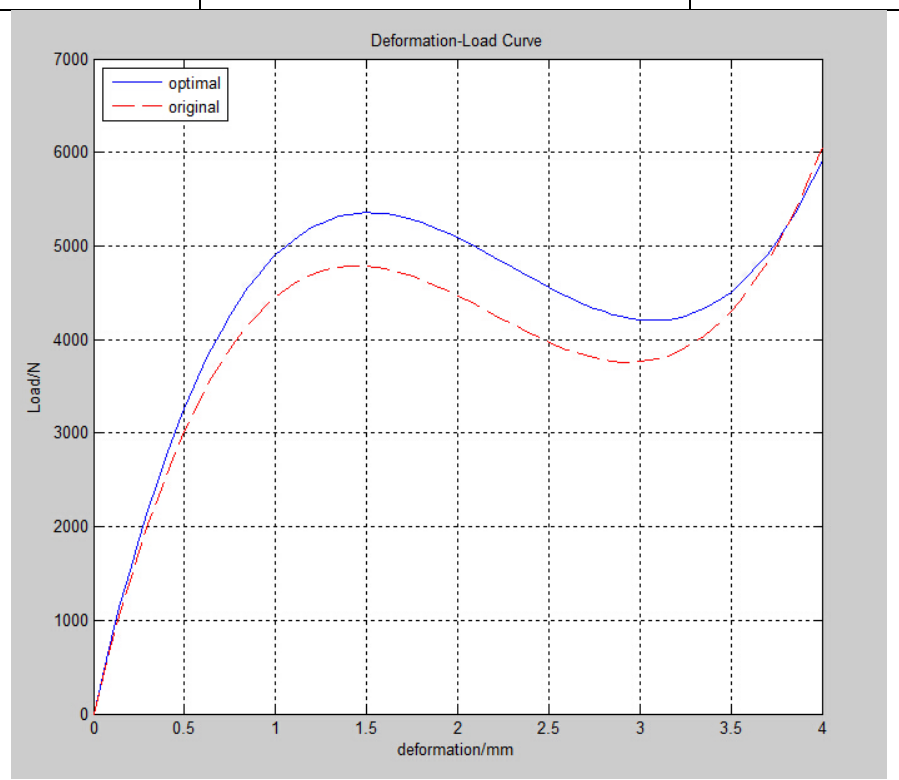

Fig 2 Structural parameters of diaphragm spring

\section{Conclusions}

In this paper, the results of the diaphragm spring could be completed rightly, with solving the multiple independent variables, and obtained simultaneously the desired effect. The idea of genetic algorithm is easy to understand, When the GA running, it emphasizes the advantages of randomly chosen for global optimization capability. It is a algorithm that easily implemented that find the optimal solution for the diaphragm spring.

\section{Acknowledgements}

Higher Education Reform of Heilongjiang Provincne Education Department Project JG2012010679; Science and Technology of Heilongjiang Provincne Project No.12541868;Natural Science Foundation of Heilongjiang Province Project No.F201334， F201336;

\section{References}

[1] Niepage P.Vergleich verschiedenen Verfahren zur Berech-nung von Tellerferdern. Draht, Bd.34,Nr5.1983:252-253.

[2] Doman, Yasunori,et al.Influence of residual stress on the load-deflection curve of diaphragm springs for automobile clutches[J].JSAE Review,2003,24(2):197-203.

[3] Pährisch,M. Influence of the manufacturing process on diaphragm springs for commercial vehicles and consideration thereof in the design phase[J]. VDI Berichte, 2006: 239-254. 
[4] Xiuli Guo, optimal Design on Diaphragm Spring of Automobile Clutch[C], optimal Design on Diaphragm Spring of Automobile Clutch, Harbin, 2009: 206-208. 